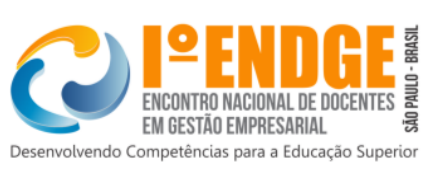

Área Temática: Gestão da Informação

\title{
A evolução das pesquisas sobre a resource-based view relacionada com a tecnologia da informação: um estudo bibliométrico
}

\author{
Henrique Takashi Adati Tomomitsu - htomomitsu@yahoo.com.br \\ Escola Politécnica da Universidade de São Paulo \\ Marcelo Dias Junqueira da Silva - marcelodj@uol.com.br \\ Escola Politécnica da Universidade de São Paulo \\ Renato de Oliveira Moraes - remo@usp.br \\ Escola Politécnica da Universidade de São Paulo
}

\begin{abstract}
Resumo
Existem várias teorias relacionadas com a gestão estratégica nas organizações, a fim de obter uma vantagem competitiva sustentável, uma que ganha cada vez mais importância nas últimas décadas é a teoria baseada em recursos de vantagem competitiva, que tem sido utilizada em vários campos de estudo. No contexto de que essa abordagem pode ser aplicada em diversos campos de estudo, o artigo tem como objetivo realizar uma revisão da literatura através de um estudo bibliométrico sobre a relação da visão baseada em recursos (RBV) com a Tecnologia da Informação (TI) para compreender seu impacto no desempenho organizacional. Através do estudo bibliométrico foi identificado que estudos ligando RBV com TI abordaram os seguintes temas: capacidades; e-business; cadeia de suprimentos; gestão estratégica; valor do negócio. Além disso, foi possível identificar um crescimento de estudos que envolvem a gestão do conhecimento, que é um recurso que é viabilizado pelos recursos de TI.
\end{abstract}

Palavras chave: tecnologia da informação, visão baseada em recurso, estudo bibliométrico.

\begin{abstract}
There are several theories related to strategic management in organizations in order to obtain a sustainable competitive advantage, one that has gained increasing importance in recent decades is the resource-based theory of competitive advantage, which has been used in various fields of study. In the context of this approach may be applicable in many fields of study, the article has as objective to conduct a review of the literature through a bibliometric study on the resource-based view (RBV) related to Information Technology (IT) to understand its impact on organizational performance. Through the bibliometric study it was identified that studies linking RBV with IT have their issues involved with: capabilities; e-business; supply chain; management strategy; business value. Moreover, it was possible to identify a growth on studies involving knowledge management which is a resource that is enabled through the use of IT resources.
\end{abstract}

Key words: information technology, resource-based view, bibliometric study. 


\section{Introdução}

Os clientes, cada vez mais exigentes, e o mercado composto por um número elevado de empresas disputando um mesmo nicho de mercado fazem com que as empresas procurem se diferenciar cada vez mais dos seus concorrentes para garantir sua continuidade no mercado. Nesse contexto a gestão estratégica tem um papel fundamental para que as organizações consigam alcançar essa diferenciação.

Existem diversas teorias relacionadas com a gestão estratégica nas organizações com o objetivo de se obter uma vantagem competitiva sustentável em relação aos seus concorrentes, uma delas que tem ganhado importância cada vez maior nas últimas décadas é a resource-based theory of competitive advantage, que tem sido empregada em diversos campos de estudos.

Essa abordagem tem um olhar para o ambiente interno das organizações, diferentemente de outras que analisam o ambiente externo, que segundo Grant (1991) durante a década de 80 era o foco da maioria dos estudos relacionados com a gestão estratégica.

Segundo Furrer et al. (2008) essa é uma abordagem estratégica a respeito da aplicação dos recursos para se atingir a vantagem competitiva, e mantém o foco sobre a associação entre os recursos que a organização possui e a performance das empresas.

No contexto de que essa abordagem pode ser aplicável em diversos campos de estudo, o artigo tem como objetivo geral realizar um estudo bibliométrico por meio de uma análise bibliométrica sobre a resource-based view sob a ótica da tecnologia da informação e seus impactos no desempenho da organização.

Por meio de uma busca preliminar na base de dados Web of Science buscando o seguinte conjunto de palavras-chaves e filtro por artigos e revisões: "Resource-Based View" and "Information Technology") OR ("Resource-Based View" and "IT capability") OR ("RBV" and "Information Technology") OR ("RBV" and "IT capability") OR ("Information Technology Capability" and "Resource-Based View") OR ("Information Technology Capability"), foi possível notar que o tema ainda tem uma importância para a literatura uma vez que nos últimos anos existe um crescimento expressivo de estudos relacionados a esse tema, conforme a Figura 1 .

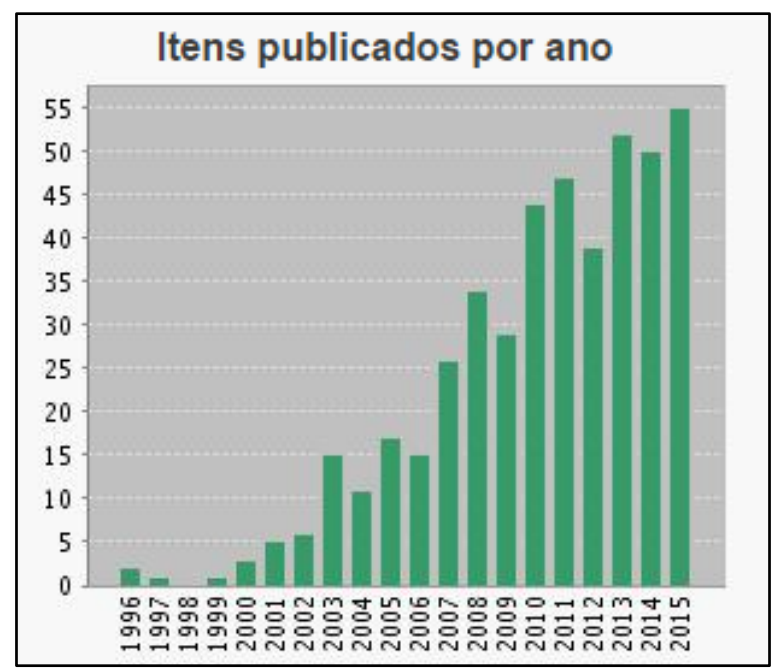

Figura 1 - Quantidade de publicações sobre o tema ao longo dos últimos 20 anos. Fonte: Web of Science $\mathrm{O}$ artigo está estruturado em 4 seções: introdução, método de pesquisa empregado para realizar o estudo, apresentação e discussão dos resultados identificados, e por fim as principais conclusões e limitações do estudo.

\section{Método de pesquisa}

A bibliometria é um método de pesquisa que tem sido amplamente utilizado por pesquisadores com o objetivo de obter um melhor entendimento sobre a literatura de um campo de estudo. 
Esse método é aplicável a qualquer campo de pesquisa, e possibilita ter uma visão geral sobre as produções científicas em um determinado campo de estudo.

Nesse contexto Subramanyam (1983) afirma que os métodos bibliométricos oferecem ferramentas de apoio para se estudar um determinado campo de pesquisa. Bakker, Groenewegen e Hond (2005) complementam que a bibliometria contribui para que seja obtido uma visão geral da estrutura de um determinado campo de estudo, o que permite identificar estruturas e padrões significativos por meio da análise de autores, periódicos, padrões de citações, dentre outros fatores.

A base escolhida para selecionar os artigos que deveriam ser analisados para realizar o estudo bibliométrico foi a ISI Web of Knowledge (Web of Science). Essa base foi escolhida devido a dois motivos principais:

- Os artigos possuem classificação pelo fator de impacto Journal Citation Report (JCR), o que permite identificar os periódicos mais relevantes.

- Os dados extraídos terem uma alta compatibilidade com o software Citespace que permitem analisar um conjunto maior de dados dos artigos. Além de possibilitar a realização de uma análise estatística descritiva sobre o conjunto de artigos selecionados, como por exemplo: distribuição de artigos publicados ao longo dos anos, distribuição de autores da amostra de artigos selecionados, distribuições de países da amostra de artigos selecionados.

O conjunto de palavras-chave usado para a busca na base ISI foi: ("Resource-Based View" and "Information Technology") OR ("Resource-Based View" and "IT capability") OR ("RBV" and "Information Technology") OR ("RBV" and "IT capability") OR ("Information Technology Capability" and "Resource-Based View") OR ("Information Technology Capability"), além do filtro para selecionar apenas artigos e revisões, o resultado dessa pesquisa foi considerado a amostra inicial para realizar a análise estatística descritiva, que consistiu nas seguintes análises:

- Distribuição de publicações de artigos ao longo dos anos: verificar a evolução do campo de estudo ao longo do tempo, além de identificação de padrões em relação as publicações e até mesmo possíveis picos em determinados períodos;

- Distribuição de publicações por autores: verificar os autores que mais publicaram ao longo dos anos, e verificar uma possível associação com sua relevância no campo de estudo;

- Distribuição de publicações por países: identificar os principais polos de desenvolvimento de estudos sobre o tema abordado;

- Distribuição de publicações por periódicos: identificar os principais periódicos relacionados com o campo de estudo;

- Classificação das principais palavras-chaves envolvidas: identificar as principais palavras chaves relacionadas como tema de estudo, o que pode auxiliar a entender possíveis tendências e/ou lacunas relacionadas com o campo de pesquisa;

- Análise de citações: identificar dentro da amostra os estudos que foram mais citados até o momento, o que pode indicar que o estudo é de alta relevância para o campo de estudo;

Em seguida foi realizado a análise de rede social sobre a amostra inicial composta pela:

- Análise de co-citações das referências: permite identificar a frequência que dois autores são citados em conjunto em outros estudos e identificar temas padrões estudados por esses autores;

Após essa busca inicial, na qual foram identificados 456 artigos, para prosseguir ao invés de se realizar mais filtros para redução do escopo dos artigos que passariam por uma análise de conteúdo, por exemplo filtro por área de pesquisa, optou-se por classificar os artigos por meio de um "Indicador de peso do artigo" calculado com base no número médio de publicações e no fator de impacto Journal Citation Report (JCR), sendo esse calculado da seguinte forma: Indicador de peso do artigo $=(1+$ "Fator de impacto $J C R$ " $)$ x Média de citações/ano A partir do cálculo do indicador de peso do artigo foi usado o software Matlab para construir o Box Plot, que é um diagrama que permite identificar os outliers dentro de uma amostra. A 
Figura 2 apresenta o diagrama encontrado, pode se notar que foram identificados 56 outliers, que vão fazer parte da amostra para realizar a análise de conteúdo.

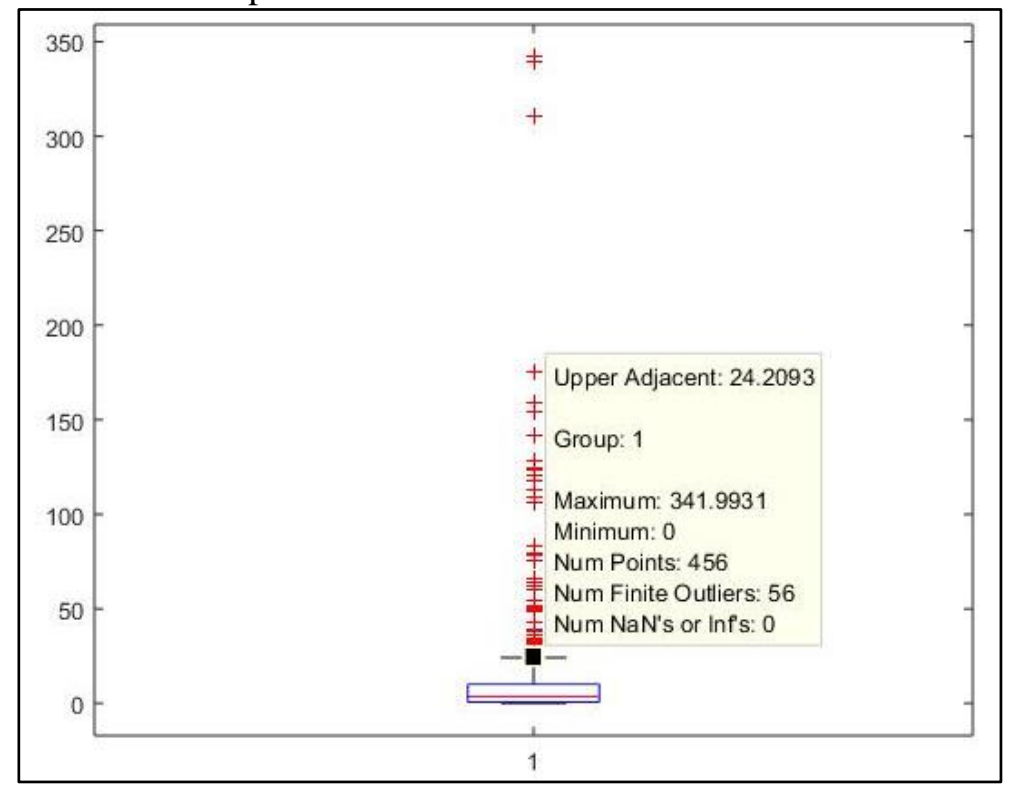

Figura 2 - Box Plot sobre o indicador de peso dos artigos. Fonte: elaborado pelos autores.

Dessa forma uma análise de conteúdo foi realizada sobre a amostra parcial, o que permite entender os principais assuntos assim como verificar os principais tipos de metodologias de pesquisa utilizadas.

\section{Análise dos Resultados}

\section{Análise das publicações}

A análise das publicações consistiu em verificar possíveis tendências de crescimento ou queda dos estudos relacionados com o tema deste artigo, conforme a Figura 1 que apresenta as publicações dos últimos 20 anos utilizadas para justificar a motivação de se realizar o estudo bibliométrico. Vale ressaltar que o primeiro artigo da amostra foi publicado em 1994. O estudo realizado por Day (1994) traz o conceito de capacidade para a gestão estratégica das organizações, destacando o uso criativo da tecnologia da informação como uma capacidade para as empresas se destacarem em relação aos seus concorrentes.

Nos últimos anos o número de publicações apresenta uma tendência de crescimento, o que implica que este campo de estudo ainda não se encontra saturado, o que indica a necessidade de mais estudos até que atinja sua estabilização. Com o objetivo de entender como os artigos estão distribuídos em relação aos autores, aos principais polos de pesquisas, e aos principais periódicos é apresentado os resultados das análises desses critérios nas Figuras 3, 4 e 5 respectivamente.

A Figura 3 apresenta a distribuição das publicações por autores, e é possível notar que existe um conjunto relevante de autores que publicaram uma quantidade representativa de artigos nesse campo de estudo, os 11 autores apresentados correspondem a 12,94 \% de todas as publicações, o que demonstra um nível representativo de centralização das publicações sobre esses autores. 


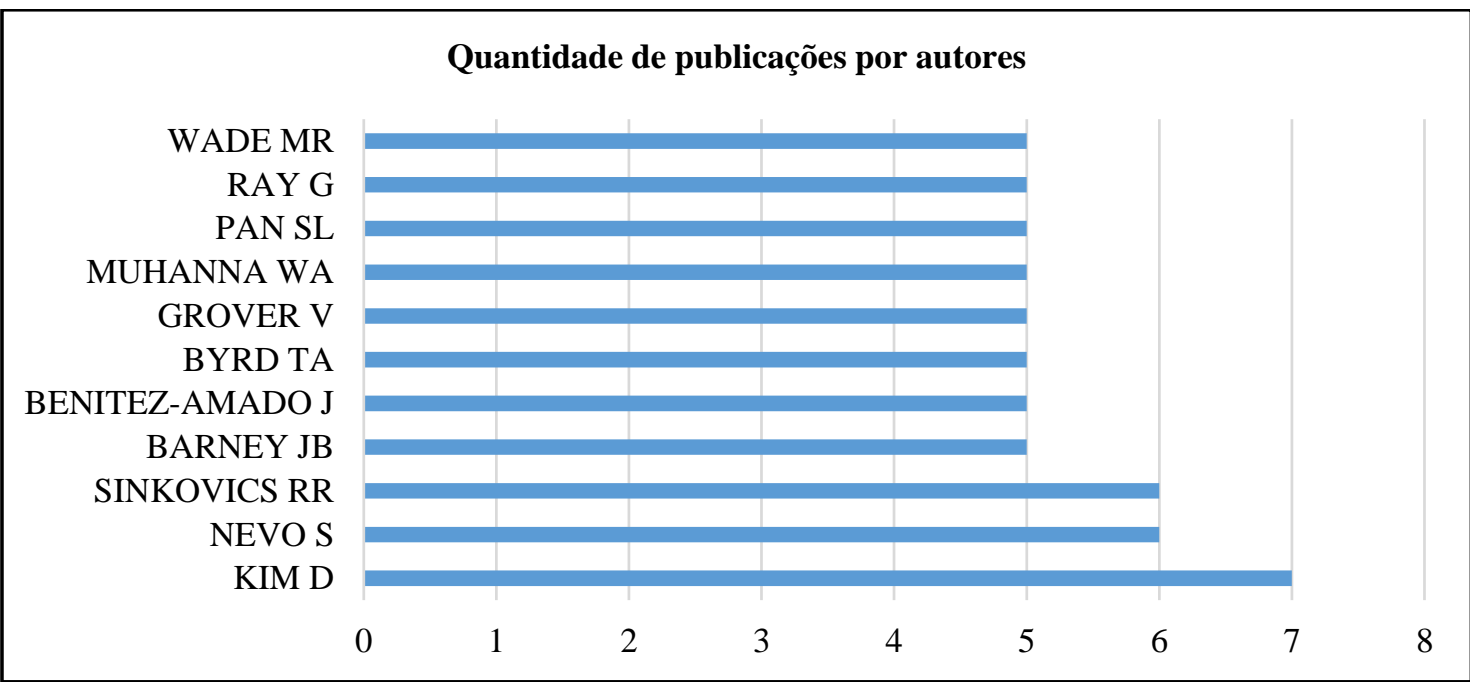

Figura 3 - Distribuição das publicações por autores. Fonte: elaborado pelos autores.

Porém é importante ressaltar que um número elevado de publicações não garante que a relevância dos seus trabalhos seja alta para o campo de estudo, para chegar a essa conclusão é preciso verificar os periódicos nos quais os artigos foram publicados e a quantidade de vezes que ele foi citado.

O principal polo de desenvolvimento de estudos sobre o assunto do presente artigo são os Estados Unidos, seguido da China, Inglaterra, Espanha e Taiwan, conforme a Figura 4. Em relação a distribuição de publicações pelo Estados Unidos é possível destacar uma estabilidade nos últimos cinco anos com uma média de 22,4 publicações por ano.

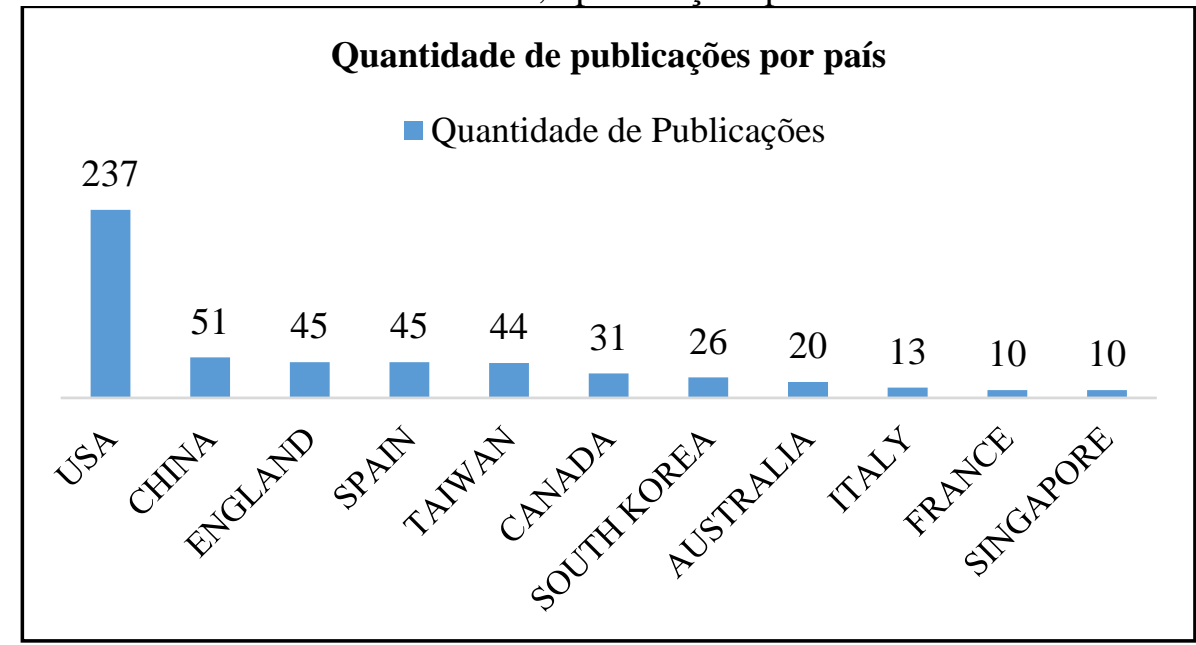

Figura 4 - Distribuição das publicações por países. Fonte: elaborado pelos autores.

Em relação a distribuição dos artigos periódico é possível destacar os periódicos MIS Quarterly, Journal of Strategic Information Systems, European Journal of Information Systems, Information Management e Industrial Management Data Systems. Isso demostra que grande parte dos estudos envolvendo a tecnologia da informação e a resource-based view são publicados em periódicos relacionados com a gestão, estratégia e sistemas de informação. 


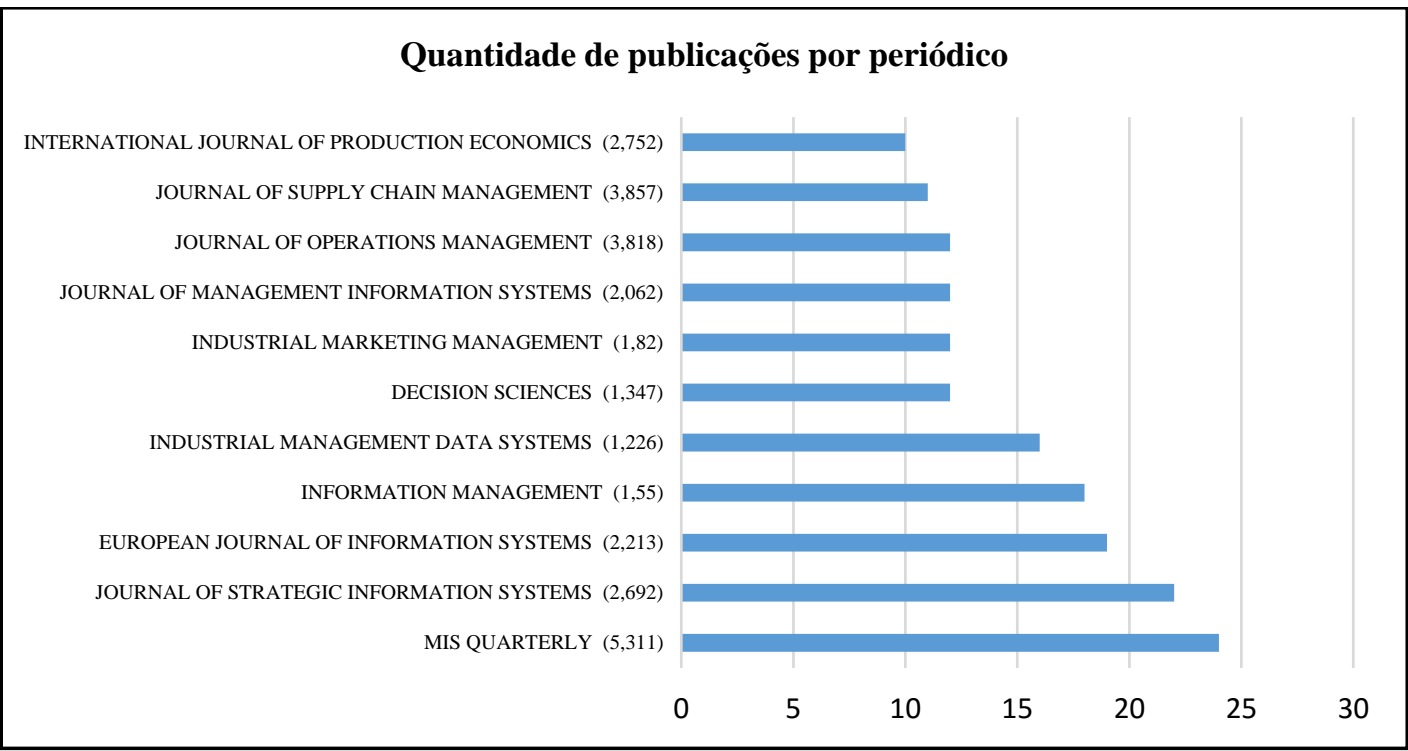

Figura 5 - Publicações por periódico e Fator de impacto. Fonte: elaborado pelos autores.

Por meio da análise das key-words dos artigos da amostra inicial foi possível notar uma alta concentração de estudos envolvendo aspectos relacionando com a performance da organização, capacidades, capacidades dinâmicas, capacidades de TI, vantagem competitiva, e-business, valor de negócio pela TI, gestão do conhecimento, gestão da cadeia de suprimento, gestão estratégica, gestão do relacionamento com o cliente, inovação e recursos de TI. A Figura 6 apresenta a consolidação das principais palavras-chaves identificadas na amostra inicial e classificadas nos seguintes campos: processos, gestão, capacidades, segmento e performance.

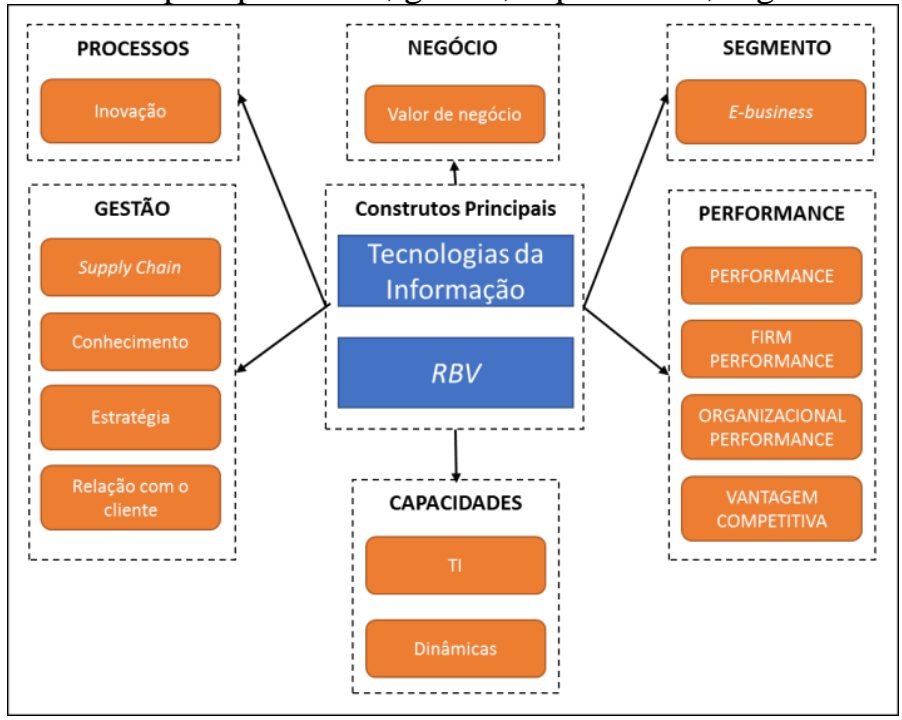

Figura 6 - Principais palavras-chaves envolvidas com a TI e RBV. Fonte: elaborado pelos autores A Tabela 1 apresenta os artigos com mais de 200 citações na amostra inicial realizada, vale notar que alguns deles não foram selecionados para a análise de conteúdo, uma vez que para a análise de conteúdo também foi levado em consideração o fator de impacto do journal. É possível notar que grande parte dos artigos foram publicados em periódicos relacionados com a gestão estratégica e com sistemas de informação.

O grande diferencial em relação ao tipo de periódico é o artigo de Day (1994) que foi publicado no Journal of Marketing que apresenta uma abordagem voltada para a questão das capacidades voltadas para a estratégia de uma organização, de forma que essa possa alcançar uma sustentabilidade no seu negócio.

Por meio da leitura dos seus abstracts é possível notar que a maioria dos estudos trazem o construto tecnologia da informação/sistemas de informação associado as seguintes temáticas: 
competência, capacidade, e-business, processos de negócio, valor de negócio, performance organizacional, vantagem competitiva.

Em relação aos tipos de métodos de pesquisa utilizados é possível notar uma certa variedade desde revisões da literatura, até a proposição de modelos que são validados por meio de pesquisas do tipo survey.

Tabela 1 - Publicações com mais citações na amostra

\begin{tabular}{|c|c|c|c|}
\hline Autores & Periódico & Ano & $\begin{array}{l}\text { Total de } \\
\text { citações }\end{array}$ \\
\hline DAY, G. S. & JOURNAL OF MARKETING & 1994 & 1514 \\
\hline BHARADWAJ, A. S. & MIS QUARTERLY & 2000 & 867 \\
\hline $\begin{array}{l}\text { MELVILLE, N.; } \\
\text { RAEMER, K.; } \\
\text { GURBAXANI, V. }\end{array}$ & MIS QUARTERLY & 2004 & 590 \\
\hline $\begin{array}{l}\text { MATA, F. J.; } \\
\text { FUERST, W. L.; } \\
\text { BARNEY, J. B. } \\
\end{array}$ & MIS QUARTERLY & 1995 & 582 \\
\hline $\begin{array}{l}\text { POWELL, T. C.; } \\
\text { DENTMICALLEF, A. }\end{array}$ & STRATEGIC MANAGEMENT JOURNAL & 1997 & 561 \\
\hline $\begin{array}{l}\text { RAY, G.; } \\
\text { BARNEY, J. B.; } \\
\text { MUHANNA, W. A. }\end{array}$ & STRATEGIC MANAGEMENT JOURNAL & 2004 & 393 \\
\hline $\begin{array}{l}\text { TIPPINS, M. J.; } \\
\text { SOHI, R. S. }\end{array}$ & STRATEGIC MANAGEMENT JOURNAL & 2003 & 361 \\
\hline NEWBERT, S. L. & STRATEGIC MANAGEMENT JOURNAL & 2007 & 319 \\
\hline $\begin{array}{l}\text { PRIEM, R. L.; } \\
\text { BUTLER, J. E. }\end{array}$ & ACADEMY OF MANAGEMENT REVIEW & 2001 & 281 \\
\hline $\begin{array}{l}\text { ZHU, K.; } \\
\text { KRAEMER, K. L. }\end{array}$ & INFORMATION SYSTEMS RESEARCH & 2005 & 267 \\
\hline $\begin{array}{l}\text { BARUA, A.; } \\
\text { KONANA, P.; } \\
\text { WHINSTON, A.B.; } \\
\text { YIN, F. }\end{array}$ & MIS QUARTERLY & 2004 & 234 \\
\hline $\begin{array}{l}\text { SANTHANAM, R.; } \\
\text { HARTONO, E. }\end{array}$ & MIS QUARTERLY & 2003 & 224 \\
\hline $\begin{array}{l}\text { ZHU, K.; } \\
\text { KRAEMER, K. L. }\end{array}$ & INFORMATION SYSTEMS RESEARCH & 2002 & 223 \\
\hline $\begin{array}{l}\text { RAY, G.; } \\
\text { MUHANNA, W. A.; } \\
\text { BARNEY, J. B. }\end{array}$ & MIS QUARTERLY & 2005 & 217 \\
\hline
\end{tabular}

Fonte: elaborado pelos autores.

Análises de rede sociais

A Figura 7 apresenta a rede de co-citação gerada pelo software CiteSpace com a seleção dos 15 autores mais citados por ano, a análise de co-citação foi realizada a partir do sobre os últimos 10 anos, de forma a verificar os principais grupos de autores no campo de estudo. Pode-se notar uma rede densa o que indica que os artigos são frequentemente citados juntos em estudos, além de ser destacado a presença de quatro clusters. 


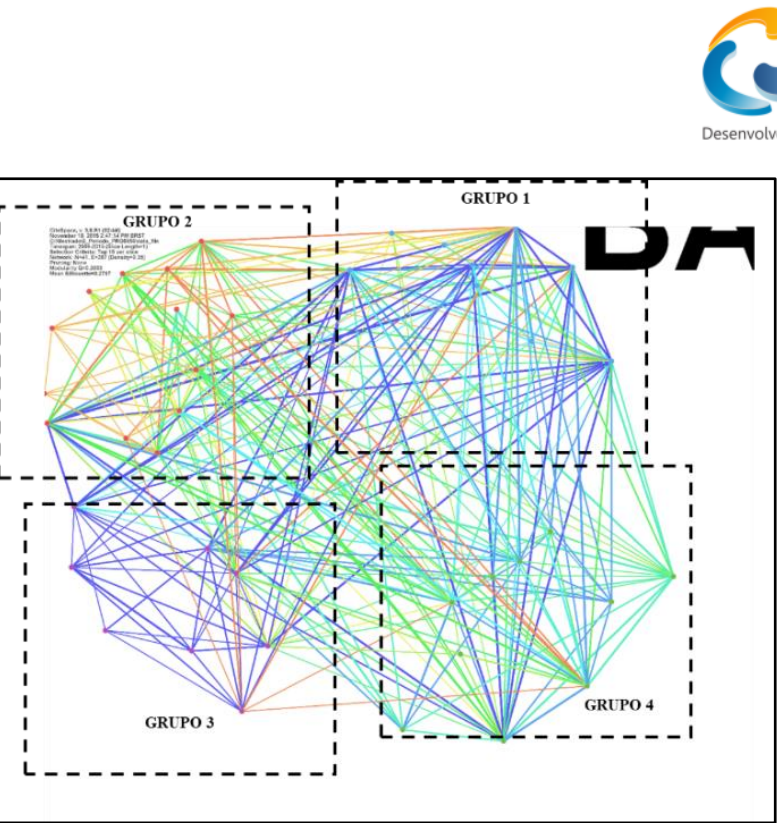

Figura 7 - Rede de co-citação. Fonte: gerado pelos autores na ferramenta CiteSpace

O grupo 1 apresenta os autores que realizam estudos associados com o uso de recursos segundo a visão baseada em recursos como um elemento estratégico que pode contribuir para que as organizações alcancem a vantagem competitiva.

Em contrapartida o grupo 2 apresenta estudos que são mais específicos relacionados a tecnologia da informação. Dessa forma é destacado o uso de recursos de TI e a forma como eles são gerenciados. A forma como esses recursos são gerenciados é definido como capacidade de TI, acredita-se que tanto os recursos quanto as capacidades de TI influenciam o desempenho organizacional.

O terceiro grupo aborda a questão das capacidades sobre a abordagem da visão baseada em recursos e sobre a teoria das capacidades dinâmicas. Alguns dos artigos abordam as capacidades mais especificamente como por exemplo a capacidade associadas a cadeia de suprimentos.

O último grupo apresenta estudos que estão relacionados principalmente com a questão de competências de TI associados a questão estratégica que pode proporcionar uma vantagem competitiva para a organização.

Pode-se notar que todos os grupos estão relacionados com impactos para as organizações seja na construção de uma vantagem competitiva ou então sobre o desempenho organizacional delas. Desta análise é possível ter conhecimento dos principais temas dos principais autores que são citados nos estudos da amostra inicial analisada.

\section{Análise de conteúdo}

A análise de conteúdo foi dividida em 3 períodos de forma a permitir uma melhor visualização da evolução do campo de estudo ao longo do tempo.

\section{Período 1 (1994 - 2005)}

$\mathrm{O}$ tema aprendizagem organizacional aparece relacionado com a RBV juntamente com a TI, Thomas et al. (2001) e Wheeler (2002) destacam que a TI tem um papel importante para promover os processos associados a aprendizagem organizacional que contribuí para um melhor desempenho organizacional.

Alguns dos artigos da amostra envolvem a RBV associada ao segmento de $e$-business que tem uma sustentação fundamental por parte dos recursos de TI. Entre os pesquisadores que tratam desse tema Zhu e Kraemer (2005) realizam uma pesquisa quantitativa por meio de uma pesquisa do tipo survey para validar o modelo que avalia a difusão e consequência do e-business a um nível empresarial levando em consideração fatores tecnológicos, organizacionais e ambientais. É destacado o valor que os recursos de TI proporcionam para o negócio.

Reforçando o fato da RBV ser uma teoria relacionada com a gestão estratégica, Priem e Butler (2001) destacam o fato de que existe um grande potencial da RBV ser usada para realizar mais contribuições para a área da gestão estratégica, destacando a TI como um possível recurso que uma organização pode usufruir nesse contexto. Hedman e Kalling (2003) destacam que a TI é um elemento que deve ser incluído em modelo de negócio, de forma que os gestores possam visualizar a relação entre eles e a estratégia de negócio. Nesse contexto Piccolli e Ives (2005) 


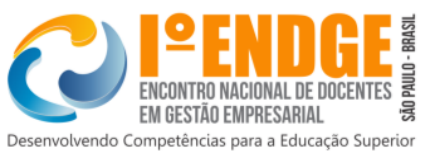

destacam que é preciso manter o foco sobre iniciativas estratégicas dependentes da TI, ou seja, aquelas que são habilitadas por meio do uso da TI.

Essas iniciativas estratégicas que são habilitados por meio do uso da TI podem ser classificadas como recursos intangíveis habilitados pela TI segundo a classificação apresentada no estudo de Bharadwaj (2000), que apresenta mais dois tipos de recursos: a infraestrutura de TI e os recursos humanos de TI. Além disso, a autora apresenta o conceito de capacidade de TI como sendo habilidade da organização reunir, integrar e gerar recursos de TI, geralmente em combinação ou co-presença com outros recursos da organização.

São inúmeros os estudos que fazem uma associação dos recursos de TI e capacidades de TI com o desempenho organizacional, o que pode contribuir para que a empresa alcance a vantagem competitiva. Em relação às metodologias mais comuns nos artigos são destacados a revisão da literatura de forma a identificar gaps ou então apresentar modelos teóricos e suas respectivas proposições, e estudos empíricos por meio da realização de estudos de caso ou surveys.

Período 2 (2006 - 2010)

O segundo período é marcado por uma quantidade expressiva de estudos envolvendo a manufatura, a logística e a cadeia de suprimentos. Banker et al. (2006) realiza uma survey e verifica que a TI apresenta efeitos indiretos significativos sobre o desempenho de uma planta de produção por meio do seu efeito sobre a capacidade dinâmica de manufatura que envolve as capacidades just-in-time (JIT) e customer and supplier participation (CSP).

Já em relação a cadeia de suprimentos Wu et al. (2006) destacam por meio de uma pesquisa survey que a TI tem um papel fundamental para a cadeia de suprimentos por permitir o intercâmbio de dados mais eficaz e uma melhor coordenação com parceiros da cadeia de suprimentos, o que pode gerar mais valor para uma organização. Além disso, é destacado que o avanço da TI e o alinhamento da TI são capazes de facilitar o desenvolvimento de capacidades da cadeia de suprimentos - intercâmbio de informação, coordenação, atividades de integração e capacidade de resposta. Dong et al. (2009), Li et al. (2009) e Paulraj et al. (2008) em seus estudos destacam o impacto positivo da TI sobre a integração da cadeia de suprimentos, e Holweg e Pil (2008) reforça a questão da TI ser um driver de vantagem competitiva por meio da alavancagem de capacidades para responder de forma mais eficiente e efetiva às mudanças ambientais.

Lai et al. (2008) foca seu estudo sobre as capacidades de TI de provedores de operações logísticas, e destaca que a capacidade de TI é um forte determinante de vantagem de custo, variedade de serviço e qualidade de serviço por meio de uma pesquisa do tipo survey.

Outro tema em destaque, nesse período, é a questão da gestão estratégia atrelada a RBV por meio do uso de tecnologias da informação. Lado et al. (2006) afirmam que a RBV pode crescer mesmo em um ambiente repleto de anomalias, paradoxos e tautologias. Nesse cenário Rivard et al. (2006) apresenta a necessidade de examinar as contribuições da TI para o desempenho organizacional por meio de um modelo que leve em consideração a complementariedade da RBV e a visão da estratégia competitiva, destacando que a TI pode desempenhar um papel importante na criação de um valor competitivo se for implementado de tal forma a se aproveitar os recursos da empresa.

Porém, é necessário um alinhamento da TI com a estratégia de negócio, e como é destacado por Oh e Pinsonneault (2007) a conquista de benefícios por meio do alinhamento da TI com a estratégia destinada a melhorar a receita é mais difícil do que a que visa à redução de custo.

Newbert (2007) realiza uma revisão da literatura e destaca que os recursos devem ser raros, valiosos, inimitáveis e não substituíveis para que a empresa possa se aproveitar de capacidades dinâmicas e de competências essenciais que podem contribuir para que a vantagem competitiva seja alcançada. Os demais estudos do período trazem a abordagem mais genérica e comum em relação a RBV e a tecnologia da informação que juntas podem impactar o desempenho da organização.

Período 3 (2011 - 2014)

Considerando a teoria de RBV, Mithas et al. (2011) concluem em seu estudo que os investimentos em TI geram mais lucratividade, ajudando o crescimento de vendas do que em 
redução de custos operacionais; em outro estudo, Mithas et al. (2012), ainda, relatam através de evidências empíricas de dados de empresas globais que estes investimentos impactam positivamente na lucratividade.

Fawcett et al. (2011) e Leuschner et al. (2013) analisando investimentos de TI, sob a ótica da cadeia de suprimentos, concluem que há influência positiva na lucratividade e desempenho da empresa. Por outro lado, Chae et al. (2014) conclui, comparando o desempenho de empresas com TI superior com outras usadas como amostra de controle que não há relação entre a capacidade de TI e o desempenho da empresa. Uma possível explicação para essa discordância é que os dados comparados pelos dois autores têm origens diferentes, no caso de Mithas, foram utilizados dados de uma conhecida empresa de pesquisa, e em Chae et al. (2014), dados da Information Week

Num estudo de caso entre um fornecedor de ERP e seus parceiros, Sarker et al. (2012) sustenta que a RBV, por si só, não é suficiente para explicar a co-criação de valor no conhecimento.

$\mathrm{Lu}$ et al. (2011) divide a capacidade de TI, construto da teoria RBV, em três dimensões: capacidade de infraestrutura de TI; capacidade de TI abrangendo negócios (parte da visão estratégica) e TI criando oportunidades de negócios (pró atividade de TI). Essa divisão ajuda na construção do conceito de agilidade organizacional, seja em capitalizar mercado ou ajustar-se operacionalmente.

Apesar de não encontrar métricas para avaliação final, Bharadwaj et al. (2013) propõe repensar a estratégia de TI, além de uma estratégia funcional, sendo alinhada, porém, não sendo subordinada à estratégia do negócio.

\section{Conclusão}

O objetivo do estudo foi verificar a evolução da relação entre a resource-based view e a tecnologia da informação ao longo os últimos anos por meio de um estudo bibliométrico. Foi possível identificar por meio das análises que esse campo de estudo ainda possui um vasto espaço para ser desenvolvido uma vez que não chegou a um ponto de saturação em relação a quantidade de estudos que são publicados sobre esse tema.

O EUA é o país que deve ser visto como uma referência em publicações sobre o tema estudado no presente estudo, visto que nos últimos anos eles mantiveram uma média elevada e constante de publicações. Em relação a distribuição de artigos por autores foi possível identificar uma concentração significativa sobre um conjunto de autores, uma vez que $12.94 \%$ das publicações se referem a apenas 11 autores.

Destaca-se que os periódicos em que os artigos da amostra se concentram são aqueles que tem um fator de impacto JCR de bastante relevância, e grande parte deles são relacionados com a gestão, estratégia e sistemas de informação.

Em relação as principais temáticas envolvidas sobre a relação da RBV com a TI é possível destacar as seguintes:

- Capacidades: é destacado que a capacidade envolve a forma como a organização utiliza os seus recursos, que pode proporcionar um melhor desempenho organizacional, e possivelmente uma vantagem competitiva sustentável caso os recursos tenham certas características essenciais - ser raro, ser valioso, ser difícil de imitar e substituir (BARNEY, 1991; NEWBERT, 2007);

- E-business: destaca-se o valor que os recursos de TI proporcionam para o negócio, com foco sobre aqueles que possibilitam esse tipo de negócio (ZHU e KRAEMER, 2005);

- Gestão estratégica: é destacado que o uso de recursos de TI pode contribuir para o valor do negócio, para tanto é preciso que exista um alinhamento entre a área de TI com as áreas de negócio, além de um maior envolvimento da alta gerência com a tomada de decisão que envolvem a TI; (PRIEM e BUTLER, 2001; HEDMAN e KALLING, 2003; PICOLLI e IVES, 2005)

- Manufatura, Logística e Cadeia de suprimentos: são diversos os estudos que destacam a importância dos recursos de TI sobre a cadeia de suprimentos, devido aos seus inúmeros benefícios com destaque para o intercâmbio de dados e a integração promovida por 
esses recursos, por meio desses benefícios é possível que a organização tenha um melhor desempenho organizacional (BANKER et al., 2006; WU et al., 2006; DONG et al., 2009; LI et al., 2009; PAULRAJ et al., 2008; LAI et al., 2008). Em contrapartida alguns autores como Chae et al. (2014) acreditam que não existe relação entre a capacidade de TI e o desempenho organizacional.

Como tendência dos estudos nos últimos anos é possível destacar um aumento em estudos relacionados com a gestão do conhecimento, que é um recurso habilitado por meio do uso da TI conforme é destacado no estudo da autora Bharadwaj (2000). Ainda existe um vasto campo de pesquisa que pode ser realizado sobre estes recursos que são habilitados pela TI, uma vez que existe uma grande diversidade desses recursos.

Uma limitação do estudo realizado é o fato de que no processo de seleção da amostra não foi realizado nenhum tipo de tratamento para identificar possíveis estudos não relacionados como tema do trabalho. Além disso na rede de co-citação poderia ter sido feito uma análise por períodos menores o que poderia contribuir mais para entender a evolução dos agrupamentos ao longo do tempo.

\section{Referências bibliográficas}

BANKER, R., BARDHAN, I., CHANG, H., LIN, S. Plant information systems, manufacturing capabilities, and plant performance. MIS Quarterly, vol. 30, n. 2, pp. 315 - 337, 2006.

BAKKER, F., GROENEWEGEN, P., HOND, F. A bibliometric analysis of 30 years of research and theory on corporate social responsibility and corporate social performance. Business \& Society, vol. 44, n. 3, pp. $283-317,2005$.

BARNEY, J., Firm Resources and Sustained Competitive Advantage. Journal of Management, vol. 17, n. 1, pp. 99-120, 1991.

BHARADWAJ, A. A resource-based perspective on information technology capability and firm performance: an empirical investigation. MIS Quarterly, vol. 24, n. 1, pp. 169 - 196, 2000.

BHARADWAJ, A., SAWY, O., PAVLOU, P., VENKATRAMAN, N. Digital business strategy: toward a next generation of insights. MIS Quarterly, vol. 37, n. 2, pp. 471 - 482, 2013.

CHAE, H., KOH, C., PRYBUTOK, V. Information technology capability and firm performance: contradictory findings and their possible causes. MIS Quarterly, vol. 38, n. 1, pp. $305-326,2000$.

DAY, G. The capabilities of market-driven organizations. Journal of Marketing, vol. 58, n. 4, pp. $37-52,1994$.

DONG, S., XU, S., ZHU, K. Information technology in supply chains: the value of IT-enabled resources under competition. Information Systems Research, vol. 20, n. 1, pp. 18 - 32, 2009.

FAWCETT, S., ALLRED, C., FAWCETT, A., MAGMAN, G. Information technology an enabler of supply chain collaboration: a dynamic-capabilities perspective. Journal of Supply Chain Management, vol. 47, n. 2, 2001.

FURRER, O., THOMAS, H., GOUSSEVSKAIA, A. The structure and evolution of strategic management field: A content analysis of 26 years of strategic management research. International Journal of Management Reviews, 2008.

GRANT, R. The Resource-Based Theory of Competitive Advantage: Implications for Strategy Formulation. California Management Review, vol. 33, pp. 114-135, 1991

HEDMAN, J., KALLING, T. The business model concept: theoretical underpinnings and empirical illustrations. European Journal of Information Systems, vol. 12, pp. 49 - 59, 2003.

HOLWEG, M., PIL, F. Theoretical perspectives on the coordination of supply chains. Journal of Operations Management, vol. 26, pp. 389 - 406, 2008. 


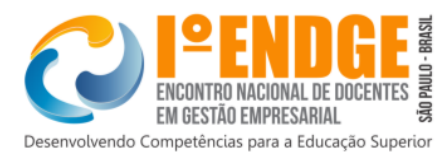

KOR, Y., MAHONEY. Edith Penrose's (1959) Contribuitions to the Resource-based View of Strategic Management. Journal of Management Studies, 2004.

LADO, A., BOYD, N., WRIGHT, P., KROLL, M. Paradox and theorizing within the resourcebased view. Adademy of Management Review, vol. 31, n. 1, pp. 115 - 131, 2006.

LAI, F., LI, D., WANG, Q., ZHAO, X. The information technology capability of third-party logistics providers: a resource-based view and empirical evidence from China. Journal of Supply Chain Management, vol. 44, n. 3, 2008.

LEUSCHNER, R., ROGERS, D., CHARVET, F. A meta-analysis of supply chain integration and firm performance. Journal of Supply Chain Management, vol. 42, n. 2, 2013.

LI, G., YANG, H., SUN, L., SOHAL, A. The impact of IT implementation on supply chain integration and performance. International Journal of Production Economics, vol. 120, pp. $125-138,2009$.

LU, Y., RAMAMURTHY, K. Understanding the link between information technology capability and organizational agility: an empirical examination. MIS Quarterly, vol. 35, n. 4, pp. $931-954,2011$.

MITHAS, S., RAMASUBBU, N., SAMBAMURTHY, V. How information management capability influences firm performance. MIS Quarterly, vol. 35, n. 1, pp. 237 - 256, 2011.

MITHAS, S., TAFTI, A., BARDHAN, I., GOH, J. Information technology and firm profitability: mechanisms and empirical evidente. MIS Quarterly, vol. 36, n. 1, pp. 205 224, 2011.

NEWBERT, S. Empirical research on the resource-based view of the firm: an assessment and suggestions for future research. Strategic Management Journal, vol. 28, pp. 121 - 146, 2007.

OH, W., PINSONNEAULT, A. On the assessment of the strategic value of information technologies. MIS Quarterly, vol. 31, n. 2, pp. 239 - 265, 2007.

PAULRAJ, A., LADO, A., CHEN, I. Inter-organizational communication as a relational competency: antecedents and performance outcomes in collaborative buyer-supplier relationships. Journal of Operations Management, vol. 26, pp. 45 - 64, 2008.

PICCOLI, G., IVES, B. Review: IT-dependent strategic initiatives and sustained competitive advantage: a review and synthesis of the literature. MIS Quarterly, vol. 29, n. 4, pp. 747776, 2005.

PRIEM, R., BUTLER, J. Is the resource-based "view" a useful perspective for strategic management research?. Academy of Management Review, vol. 26, n. 1, pp. 22 - 40, 2001.

RIVARD, S., RAYMOND, L., VERREAUTL, D. Resource-based view and competitive strategy: an integrated model of the contribution of information technology to firm performance. Journal of Information Systems, vol. 15, pp. 29 - 50, 2006.

RUGMAN, A., VERBEKE, A. Edith Penrose's contribution to the resource-based view of strategic management. Strategic Management Journal, vol. 23, pp. 769-780, 2002.

SARKER, S., SARKER, S., SAHAYM, A., ANDERSEN, N. Exploring value cocreation in relationships between an ERP vendor and its partners: a revelatory case study. MIS Quarterly, vol. 36, n. 1, pp. 317 - 338, 2012.

SUBRAMANYAM, K. Bibliometric studies of research collaboration: a review. Journal of Information Science, pp. 33-38, 1983.

THOMAS, J., SUSSMAN, S., HENDERSON, J. Understanding “strategic learning”: linking organizational learning, knowledge management, and sensemaking. Organization Science, vol. 12, n. 3, pp. $331-345,2001$.

WERNERFELT, B. A resource-based view of the firm. Strategic Management Journal, vol. 5, pp. 171-180, 1984. 
WHEELER, B. NEBIC: A dynamic capabilities theory of for assessing net-enabler. Information Systems Research, vol. 13, n. 2, pp. 125 - 146, 2002.

WU, F., YENIYURT, S., KIM, D., CAVUSGIL, T. The impact of information technology on supply chain capabilities and firm performance: a resource-based view. Industrial Marketing Management, vol. 35, pp. 493 - 504, 2006.

ZHU, K., KRAEMER, K. Post-adoption variations in usage and value of e-business by organizations: cross-country evidence from the retail industry. Information Systems Research, vol. 16, n. 1, pp. $61-84,2005$. 